\title{
Caracterización Fisicoquímica de Emulsiones Aceite/Agua a partir de Uchuva (Physalis peruviana) como Ingrediente para la Industria Alimenticia
}

\author{
Natalia Ramírez-Nieto(1), Yolima Baena ${ }^{(2)}$ y Coralia Osorio(3)* \\ (1) Universidad Nacional de Colombia-Sede Bogotá, Facultad de Ingeniería, Departamento de Ingeniería Química y \\ Ambiental, Bogotá, Colombia (e-mail: nramirezn@unal.edu.co), \\ (2) Universidad Nacional de Colombia- Sede Bogotá, Facultad de Ciencias, Departamento de Farmacia, Bogotá, \\ Colombia (e-mail: ybaenaa@unal.edu.co) \\ (3) Universidad Nacional de Colombia- Sede Bogotá, Facultad de Ciencias, Departamento de Química, AA 14490, \\ Bogotá, Colombia (e-mail: cosorior@unal.edu.co)
}

Recibido Oct. 22, 2018; Aceptado Dic. 21, 2018; Versión final Feb. 19, 2019, Publicado Jun. 2019

\begin{abstract}
Resumen
Con el objeto de utilizar los ingredientes biofuncionales de la uchuva (Physalis peruviana), se prepararon emulsiones aceite/agua $(\mathrm{O} / \mathrm{W})$ usando como fase oleosa, el extracto de uchuva obtenido con aceite de girasol y asistido con ultrasonido. Las emulsiones se obtuvieron espontáneamente utilizando diferentes proporciones de fase oleosa/fase acuosa. Se usó Tween 20 como surfactante, propilenglicol como co-surfactante y goma xantana como viscosante. Las emulsiones se caracterizaron fisicoquímicamente, midiendo $\mathrm{pH}$, conductividad, distribución de tamaño de gota, y potencial Z. También se realizó la medición de contenido de carotenoides y color mediante colorimetría triestímulo. Las emulsiones obtenidas con adición de goma xantana presentaron un tamaño de gota menor a $3.43 \mu \mathrm{m}$, incrementando así su estabilidad. Las emulsiones con bajo contenido de fase oleosa se identificaron como las más estables por la medición de potencial Z. Sin embargo, en la fase oleosa se comprobó la actividad antihipercolerestolémica frente a la inhibición de la HMG-CoA reductasa, con valores mayores que el control pravastatina. Esto confirma el potencial de las emulsiones de uchuva como ingrediente biofuncional alimentario.
\end{abstract}

Palabras clave: emulsiones; extracción asistida con ultrasonido; carotenoides; actividad hipocolerestolémica

\section{Physicochemical Characterization of O/W Emulsions from Goldenberry (Physalis peruviana) as an Ingredient for Food Industry}

\begin{abstract}
With the aim of using the biofunctional ingredients of goldenberry (Physalis peruviana), oil/water emulsions $(\mathrm{O} / \mathrm{W})$ were prepared by using as the oily phase sunflower oil fruit pulp extract (ultrasound assisted). Emulsions were spontaneously obtained, with different amount of oil phase/aqueous phase. Also,Tween 20 was employed as surfactant, propylene glycol as cosurfactant, and xanthan gum as viscosity increasing agent. Physicochemical characterization of the emulsions was carried out by measuring $\mathrm{pH}$, conductivity, droplet size distribution and Z potential. Also, carotenoid content and color (tristimulus colorimetry) were determined. The emulsions obtained with addition of xanthan gum showed a droplet size lower than $3.43 \mu \mathrm{m}$, increasing their stability. The emulsions containing lower amount of oily phase, were the most stable based on their $Z$ potential values. However, the oily phase showed a high anti hypercholesterolemic activity under the assay of HMG$\mathrm{CoA}$ reductase inhibition, exhibiting higher values than the control pravastatin. This confirms the potential of Goldenberry emulsions as biofunctional food ingredient.
\end{abstract}

Keywords: emulsions; ultrasound assisted extraction; carotenoids; hypocholesterolemic activity 


\section{INTRODUCCIÓN}

Colombia es uno de los principales productores de uchuva a nivel mundial; su producción se ha centrado en los departamentos de Antioquia, Boyacá y Cundinamarca, los cuales aportan el 92,84\% de la producción nacional, reportando un valor de 13260 toneladas para el año 2014 (Agronet, 2018). Este producto se exporta principalmente a la Unión Europea, con una cifra de exportación de 5.197 toneladas netas en 2016. Los principales destinos de exportación son Holanda (64.3\%), Alemania (16.2\%) y Estados Unidos (5.2\%) (Analdex, 2018). Generalmente esta fruta se consume en fresco. Es una fuente excelente de provitamina A, vitamina $\mathrm{C}$, complejo de vitamina $\mathrm{B}$ y minerales. Adicionalmente, esta fruta, sometida a un proceso de deshidratación, ha sido incluida de manera exitosa en el mercado de Estados Unidos y ha sido catalogada como una "superfruit" (término de mercadeo utilizado en la industria de alimentos y bebidas para describir frutas que presentan características sensoriales excepcionales y beneficios para la salud) gracias a sus propiedades biofuncionales (Osorio et al., 2016). Sin embargo, el comercio de la uchuva actualmente enfrenta un reto en cuanto a su producción, ya que existen dificultades por parte de los productores para comercializar la fruta que no cumple los exigentes criterios de exportación, lo cual conlleva a una pérdida cercana al $40 \%$ de la producción total.

En la actualidad, el desarrollo de nuevos productos alimenticios tiene como objetivo no sólo proveer los nutrientes necesarios para la subsistencia, sino prevenir enfermedades relacionadas con la dieta y mejorar la salud física y mental de los consumidores constituyéndose así en alimentos funcionales (Siró et al., 2008). El número de estudios sobre la identificación de nuevos ingredientes de fuentes naturales, con el propósito de ser incorporados en un alimento funcional, se ha incrementado, tanto así que se ha creado una nueva área de investigación en la Ciencia y Tecnología de Alimentos para promover el bienestar del consumidor (Vieira da Silva et al., 2016). A la fecha se han desarrollado y caracterizado productos derivados de la uchuva, como jugo pasteurizado (Ramadan et al., 2013), granulados enriquecidos en aroma y color (Ramírez, 2009), jugo clarificado (Giraldo et al. 2017) y formulaciones en polvo utilizando secado por atomización con aire caliente (Bernal, et al., 2016), entre otros. En el jugo de uchuva se ha evaluado su actividad hipocolerestolémica in vivo (Ramadan et al., 2013) y se conoce que varios compuestos liposolubles como esteroles, vitamina $E, y$ carotenoides presentes en la uchuva, son responsables de sus características biofuncionales. Así, en este trabajo, se desarrollaron y caracterizaron emulsiones de uchuva, conteniendo fitoquímicos de diferente polaridad, con miras a obtener un ingrediente líquido que pueda usarse en la industria alimenticia.

\section{MATERIALES Y MÉTODOS}

Materiales: Polisorbato 20 (Tween 20, AppliChem Panreac ITW), aceite de girasol (grado comercial), propilenglicol (Ciacomeq SAS, Bogotá-Colombia), goma xantana (grado alimenticio), beta caroteno $95 \%$ (Sigma-Aldrich, St. Louis, MO, USA) y cloruro de potasio (KCl) (Merck) para la calibración del conductímetro.

Obtención del extracto de uchuva: Se compraron frutos de uchuva (Physalis peruviana) en los mercados locales de Bogotá, en un estado de madurez entre 3 y 4 de acuerdo con la norma técnica colombiana NTC 4580 , caracterizados por un contenido de sólidos solubles de 14.1 y $14.5^{\circ}$ Brix, respectivamente, y un rango máximo de acidez entre $2.03-2.34 \%$, expresado como ácido cítrico (ICONTEC, 1999). Posteriormente, las uchuvas se cortaron en tajadas delgadas y homogéneas (aprox. $3 \mathrm{~mm}$ de espesor), luego se congelaron a $70^{\circ} \mathrm{C}$ por 24 horas y se secaron en un liofilizador (Beta1-8 LD plus, Martin Christ, Alemania), el cual funciona en dos etapas, la primera a $0.1 \mathrm{Atm},-56^{\circ} \mathrm{C}$ por 20 horas, y la segunda a $0.01 \mathrm{Atm}$ y $-77^{\circ} \mathrm{C}$ por 4 horas. Las frutas secas se pulverizaron antes de iniciar el proceso de extracción asistida por ultrasonido (EAU), el cual se adaptó del usado por Martínez (2016). En cada ensayo se utilizaron $4.6 \mathrm{~g}$ de uchuva liofilizada y pulverizada, los cuales se mezclaron con $30 \mathrm{~mL}$ de aceite de girasol, el cual se sumergió en un baño de ultrasonido con potencial nominal total de $425 \mathrm{~W}$, manteniendo la temperatura por debajo de $40^{\circ} \mathrm{C}$. El extracto se filtró antes de preparar las emulsiones.

Formulación de las emulsiones: Se utilizó el método de emulsificación espontánea (Komaiko y McClements, 2016), con el extracto de uchuva en aceite de girasol como fase oleosa y agua destilada como fase acuosa, Tween 20 como surfactante y propilenglicol (PG) como co-surfactante, variando las proporciones de los ingredientes mencionados anteriormente de acuerdo con el diseño presentado en la Tabla 1. En cada ensayo, la fase oleosa se mezcló primero con el surfactante con agitación suave y luego la mezcla se homogenizó con el PG; posteriormente se adicionó la fase acuosa gota a gota con agitación manual hasta completa homogenización. En los ensayos que tenían goma xantana, ésta se dispersó previamente en la fase acuosa antes de adicionarla al resto de la emulsión.

Caracterización fisicoquímica de las emulsiones: Se midió el pH de las emulsiones usando un pHmetro 370 (Jenway, Londres, UK) y la conductividad eléctrica usando un conductímetro portátil modelo 470 (Jenway, Londres, UK) calibrado con solución $0.01 \mathrm{M}$ de KCl, con conductividad específica de $1413 \mu \mathrm{S}$ a $18{ }^{\circ} \mathrm{C}$ (Ghosh 
et al., 2013). Todas las mediciones se realizaron por triplicado reportando el promedio y su desviación estándar. La viscosidad de las emulsiones se midió utilizando un viscosímetro rotacional (Thermo Scientific HAAKE Viscotester 550), en donde se realizó la medición del esfuerzo cortante variando la deformación para 150 puntos por cada emulsión. La viscosidad en Pa.s, se determinó como la pendiente de la recta obtenida al graficar el esfuerzo cortante vs. la deformación. La distribución del tamaño de gota se midió utilizando la técnica de DLS (Dynamic Light Scattering) en un equipo Mastersizer 3000 (Malvern Instruments Ltd., Londres, $\mathrm{UK})$.

Tabla 1: Diseño experimental para la preparación de emulsiones O/W de uchuva.

\begin{tabular}{|c|c|c|c|c|c|}
\hline Muestra & Fase acuosa (\%) & Fase oleosa (\%) & Tween 20 (\%) & $P G(\%)$ & $\begin{array}{c}\text { Goma xantana (\%) } \\
\text { (porcentaje de fase acuosa) }\end{array}$ \\
\hline M1 & 60 & 5 & 30 & 5 & 0 \\
M2 & 60 & 15 & 20 & 5 & 0 \\
M3 & 60 & 25 & 10 & 5 & 0 \\
M4 & 70 & 5 & 20 & 5 & 0 \\
M5 & 80 & 5 & 10 & 5 & 0 \\
M6 & 50 & 30 & 5 & 0 \\
M7 & 62 & 3 & 30 & 5 & 0 \\
M8 & 58 & 30 & 5 & 0 \\
M9 & 65 & 5 & 25 & 5 & 0 \\
M10 & 60 & 10 & 25 & 5 & 0 \\
\hline M1A & 60 & 5 & 30 & 5 & 0.17 \\
M1B & 60 & 5 & 30 & 5 & 0.33 \\
M6A & 50 & 15 & 30 & 5 & 0.17 \\
M6B & 50 & 15 & 30 & 5 & 0.17 \\
M10A & 60 & 10 & 25 & 5 & 0.33 \\
M10B & 60 & 10 & 25 & 5 & \\
\hline
\end{tabular}

La medición del potencial $Z$ se realizó únicamente para las emulsiones con goma xantana. Para al fin, se realizó una dilución de cada muestra en proporción 1/1000, en una solución de $\mathrm{NaCl} 0.1 \mathrm{mM}$. Posteriormente se introdujo la cantidad necesaria de muestra en el recipiente correspondiente y se realizó la medición cinco (5) veces para cada muestra. Las medidas fueron realizadas utilizando un equipo Zetasizer Nano (Malvern Instruments Ltd., Londres, UK). La composición del medio dispersante en todos los casos fue agua con una constante dieléctrica de 78.5 , viscosidad de $0.8872 \mathrm{cP}$ e índice de refracción de 1.330 , todo a temperatura constante de $25^{\circ} \mathrm{C}$. La medida objetiva del color se realizó en un equipo Varian Cary 5000 UV-Vis-NIR (Varian, Australia) equipado con lámpara de Tungsteno. Se midió la transmitancia en un rango de 830 a $360 \mathrm{~nm}$ tomando datos a una velocidad de barrido $600 \mathrm{~nm} /$ minuto. Los datos fueron convertidos a los parámetros de colorimetría triestimulo $L^{*}, a^{*}, b^{*}$ mediante el software Cary WinUV. La determinación del contenido de carotenoides totales se realizó por espectrofotometría UV/VIS siguiendo el método propuesto por Wrolstad et al., (2005). Como estándar se utilizó $\beta$-caroteno disuelto en $n$-hexano (Merck, Darmstadt, Alemania). Se midió la absorbancia de soluciones de concentración conocida en un espectrofotómetro Jenway (modelo 7305, Londres, UK) a una $\lambda$ de $450 \mathrm{~nm}$. Las muestras se evaluaron de la misma forma y la cantidad de carotenoides se expresó como $\mathrm{mg}$ de $\beta$ caroteno $/ \mathrm{mL}$ de emulsión.

Determinación de la actividad hipocolerestolémica: La actividad hipocolorestelómica de la fase acuosa y fase oleosa de uchuva, se midió con el kit enzimático de HMG-CoA reductasa (Sigma-Aldrich, St. Louis, MO, USA). Se siguió el protocolo proporcionado por el fabricante. La concentración de la solución stock de HMG-CoA reductasa fue de $0.5-0.75 \mathrm{mg} / \mathrm{mL}$. Cada extracto se adicionó a una mezcla de reacción que contenía el substrato de HMG-CoA $(400 \mu \mathrm{M})$, y una solución buffer de fosfato de sodio (100 mM, pH 7.4) seguido de la adición de la HMG-CoA reductasa $(2 \mu \mathrm{L})$. La reacción se incubó a $37^{\circ} \mathrm{C}$ y se midió la absorbancia a $\lambda=340 \mathrm{~nm}$ durante 15 minutos en un lector multimodal de microplacas Berthold (Alemania). Se usó pravastatina (SigmaAldrich Co.) como control positivo.

\section{RESULTADOS Y DISCUSIÓN}

En la Tabla 1 se resumen las diferentes emulsiones preparadas, teniendo en cuenta que entre más bajo sea el porcentaje de fase oleosa y entre más alta sea la relación surfactante:fase oleosa, mayor probabilidad de obtener microemulsiones existe, las cuales son muy apetecidas por la industria por su transparencia. Sin embargo, en este caso, el porcentaje de fase oleosa no debía ser muy bajo ya que es la fase que contiene los fitoquímicos bioactivos. El Tween 20 se usó como surfactante debido a su alto balance hidrofílico-lipofílico (HLB), el cual es favorable para la formación de emulsiones O/W; y se seleccionó PG como co-surfactante debido a que incrementa el carácter hidrofílico del surfactante (Abd-Allah et al., 2010), ayudando así a disminuir la tensión interfacial agua-aceite. 
Cualitativamente se evaluó la estabilidad de M1-M10, midiendo el tiempo en el cual se rompía la emulsión (formación de dos fases). Así, se seleccionaron las emulsiones M1, M6 y M10 por ser las más estables, y se decidió incluir en la formulación goma xantana como viscosante para incrementar su estabilidad, puesto que de esta manera se ve disminuida la velocidad de movimiento de las gotas de la fase dispersa y por consiguiente la posibilidad de agregación y posterior coalescencia, como etapas previas a la separación de las fases (Schramm, 2005). La composición de goma xantana se definió haciendo variaciones entre 0.1 y $0.5 \%$ (sobre la fase acuosa). Se observó un incremento significativo de la viscosidad del sistema con $0.5 \%$ de goma xantana; por esta razón, sólo se seleccionaron los porcentajes $0.17 \%$ y $0.33 \%$ debido a que incrementaron la estabilidad sin modificar drásticamente la viscosidad de la emulsión final. La adición de la goma xantana permitió incrementar la estabilidad considerablemente (más de 8 días).

\section{Caracterización fisicoquímica de las emulsiones}

La caracterización fisicoquímica de todas las emulsiones se realizó a $18{ }^{\circ} \mathrm{C}$. Los resultados de $\mathrm{pH}$, conductividad, viscosidad y tamaño de gota se presentan en la Tabla 2.

Tabla 2: Caracterización fisicoquímica de las emulsiones $\mathrm{O} / \mathrm{W}$ de uchuva a $18^{\circ} \mathrm{C}$. Letras iguales (a-f) en la misma columna significa que no hay diferencias significativas $(p<0.5)$. *La composición de cada emulsión se presenta en la Tabla 1. $\mathrm{NN}=$ No newtoniano

\begin{tabular}{|c|c|c|c|c|}
\hline Muestra & Conductividad $(\mu \mathrm{s})$ & $p H$ & $\begin{array}{c}\text { Viscosidad } \\
\text { calculada }(\text { Pa.s })\end{array}$ & $\begin{array}{c}\text { Tamaño de } \\
\text { gota }(\mu \mathrm{m})\end{array}$ \\
\hline M1 & $112.2 \pm 4.9^{\mathrm{a}}$ & $7.65 \pm 0.15^{\mathrm{a}, \mathrm{c}}$ & $0.0314^{\mathrm{d}}$ & $6.35^{\mathrm{b}}$ \\
M2 & $104.4 \pm 3.4^{\mathrm{a}, \mathrm{c}}$ & $7.43 \pm 0.13^{\mathrm{a}, \mathrm{c}, \mathrm{d}}$ & $0.0158^{\mathrm{b}}$ & $31.10^{\mathrm{e}}$ \\
M3 & $82.6 \pm 4.7^{\mathrm{b}}$ & $7.06 \pm 0.05^{\mathrm{b}}$ & $0.0069^{\mathrm{a}}$ & $59.50^{\mathrm{f}}$ \\
M4 & $103.6 \pm 2.8^{\mathrm{c}}$ & $7.51 \pm 0.21^{\mathrm{a}, \mathrm{c}}$ & $0.0098^{\mathrm{a}}$ & $17.60^{\mathrm{d}}$ \\
M5 & $74.2 \pm 0.4^{\mathrm{d}}$ & $7.37 \pm 0.10^{\mathrm{a}, \mathrm{d}}$ & $0.0040^{\mathrm{a}}$ & $30.20^{\mathrm{e}}$ \\
M6 & $91.0 \pm 2.7^{\mathrm{e}}$ & $7.66 \pm 0.12^{\mathrm{c}}$ & $0.0646^{\mathrm{b}}$ & $10.30^{\mathrm{c}}$ \\
M7 & $130.7 \pm 0.4^{\mathrm{f}}$ & $7.33 \pm 0.09^{\mathrm{a}, \mathrm{d}}$ & $0,0256^{\mathrm{c}}$ & $12.30^{\mathrm{c}}$ \\
M8 & $107.6 \pm 3.0^{\mathrm{a}}$ & $7.56 \pm 0.10^{\mathrm{a}}$ & $0.0381^{\mathrm{e}}$ & $9.09^{\mathrm{c}}$ \\
M9 & $102.7 \pm 1.8^{\mathrm{c}}$ & $7.42 \pm 0.02^{\mathrm{d}}$ & $0.0213^{\mathrm{c}}$ & $11.60^{\mathrm{c}}$ \\
M10 & $99.2 \pm 1.8^{\mathrm{c}}$ & $7.40 \pm 0.04^{\mathrm{d}}$ & $0.0230^{\mathrm{c}}$ & $13.00^{\mathrm{c}}$ \\
\hline M1A & $163.2 \pm 2.3^{\mathrm{g}}$ & $7.21 \pm 0.11^{\mathrm{b}}$ & $\mathrm{NN}$ & $2.60^{\mathrm{a}}$ \\
M1B & $179.7 \pm 0.6^{\mathrm{h}}$ & $7.08 \pm 0.09^{\mathrm{b}}$ & $\mathrm{NN}$ & $2.96^{\mathrm{a}}$ \\
M6A & $93.0 \pm 1.9^{\mathrm{e}}$ & $7.07 \pm 0.08^{\mathrm{b}}$ & $\mathrm{NN}$ & $2.80^{\mathrm{a}}$ \\
M6B & $116.5 \pm 0.4^{\mathrm{a}}$ & $7.19 \pm 0.11^{\mathrm{b}}$ & $\mathrm{NN}$ & $3.43^{\mathrm{a}}$ \\
M10A & $120.5 \pm 0.5^{\mathrm{i}}$ & $7.26 \pm 0.05^{\mathrm{d}}$ & $\mathrm{NN}$ & $2.68^{\mathrm{a}}$ \\
M10B & $202.7 \pm 2.3^{\mathrm{j}}$ & $7.12 \pm 0.10^{\mathrm{b}}$ & $\mathrm{NN}$ & $2.71^{\mathrm{a}}$ \\
\hline
\end{tabular}

Los valores de $\mathrm{pH}$ obtenidos variaron en un rango entre 7.06 a 7.66 debido a la presencia de Tween 20 , a pesar de las variaciones en composición de los ingredientes de la emulsión y a la acidez de la uchuva. Adicionalmente, los valores de conductividad variaron de 74.2 a $202.7 \mu \mathrm{S}$ a $18{ }^{\circ} \mathrm{C}$. Analizando los datos de conductividad para los ensayos M1, M6, M7 y M8, en los cuales la composición de Tween 20 fue constante, se evidencia una relación directa con la proporción agua/aceite, pues una mayor proporción de agua permite una mayor movilidad de los iones y por tanto mayor conductividad. En la Figura 1 se muestra la gráfica de conductividad vs. el porcentaje de goma xantana para cada serie de emulsiones con composición de FA, FO, Tween 20 y PG constantes.

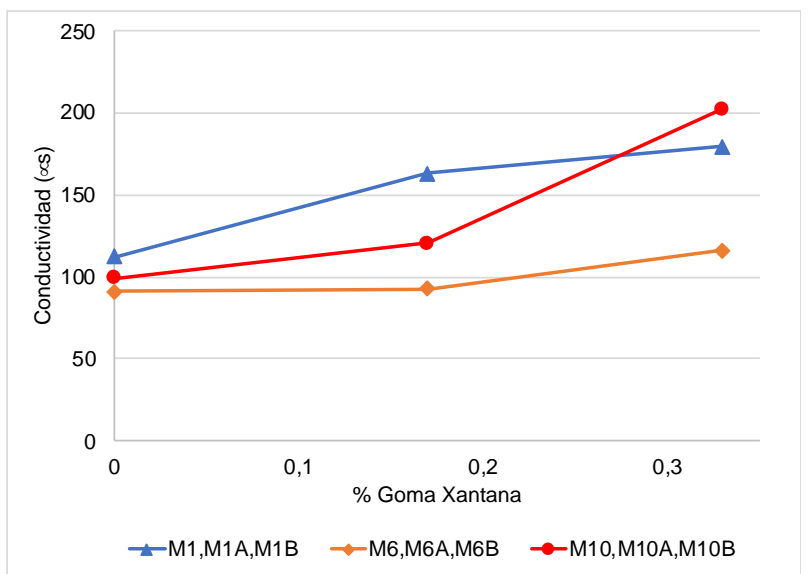

Fig. 1: Variación de la conductividad de las emulsiones $\mathrm{O} / \mathrm{W}$ de uchuva vs. \% de goma xantana (expresada como \% de FA). 
Considerando las tendencias de esta figura, se observa que la conductividad de las emulsiones aumenta al incrementar la concentración de goma xantana debido al incremento de la movilidad de las moléculas de goma xantana en la fase acuosa de la emulsión (El-Ashhab et al., 2008). La conductividad de los polielectrolitos como la goma xantana depende del número de iones por unidad de volumen y su movilidad en solución, lo que es característico de una emulsión O/W, como las obtenidas. Por esta razón, para cada muestra que contiene goma xantana, el valor de conductividad mayor en comparación a la que no tiene goma xantana, está dado por los residuos de los ácidos glucurónico y pirúvico (-COO-) presentes en la estructura de la goma xantana y sus contraiones tales como $\mathrm{Na}^{+}, \mathrm{K}^{+}, \mathrm{Ca}^{2+}$ y Mg${ }^{2+}$ (Brunchi et al., 2016).

La influencia de la concentración de la fase oleosa en las propiedades reológicas de las emulsiones se estimó realizando las mediciones de viscosidad a $21^{\circ} \mathrm{C}$ (Tabla 2). Los resultados al hacer las medidas de viscosidad de las emulsiones sin y con goma xantana se muestran en las Figuras $2 \mathrm{~A}$ y $2 \mathrm{~B}$, respectivamente. La viscosidad aparente se muestra en función de la velocidad de deformación. Todas las emulsiones sin goma xantana se comportaron como fluidos newtonianos; su viscosidad no cambió con el aumento de la velocidad de deformación (Dluzewska et al., 2006). En contraste, las emulsiones que contenían goma xantana presentaron comportamiento de fluido no newtoniano, independientemente de la composición de fase oleosa; este comportamiento es el esperado al usar gomas como estabilizantes en la fase acuosa (Dluzewska et al., 2006).

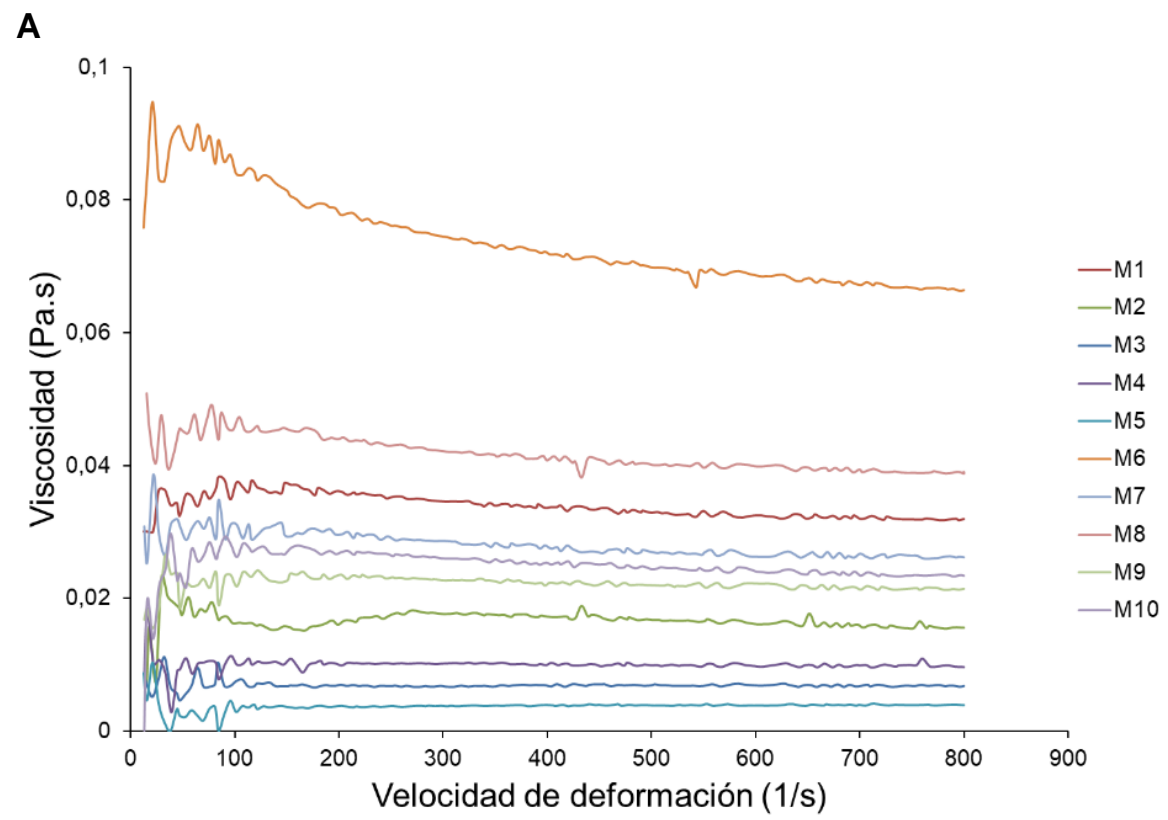

B

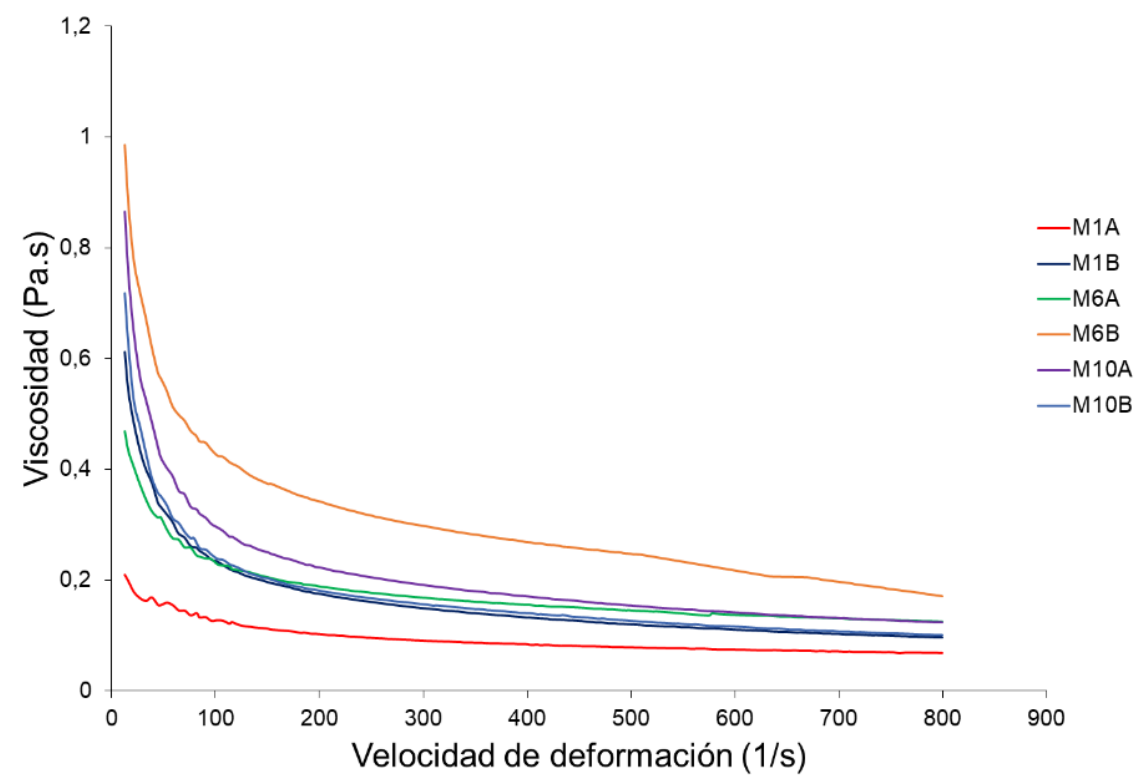

Fig. 2: Viscosidad de las emulsiones $\mathrm{O} / \mathrm{W}$ de uchuva (A) $\sin y(\mathrm{~B})$ con goma xantana a $21^{\circ} \mathrm{C}$. 
Se analizó la influencia del incremento de la relación aceite/agua en la viscosidad para las emulsiones sin goma xantana que tienen composición de Tween 20 constante de 30\% (M1, M6, M7 y M8). Se observó que la viscosidad presenta un incremento significativo al incrementar la relación aceite/agua, obteniendo la viscosidad más alta para la emulsión M6, que tenía la concentración más alta de fase oleosa. El incremento de viscosidad de las emulsiones relacionado con el incremento de la concentración de la fase dispersa corresponde a lo reportado en la literatura (Dluzewska et al., 2006). Algunas investigaciones sobre las propiedades reológicas de las emulsiones han mostrado que uno de los factores más influyentes en su viscosidad, es la fracción de volumen de la fase dispersa $(\varphi)$ la cual es igual al volumen de la fase oleosa dispersa en gotas sobre el volumen total de la emulsión (McClements, 2005). Se encontró también que el incremento en la relación Tween 20/agua, influye en la viscosidad de las emulsiones (M1, M2, M3 y M10), haciendo que su valor también aumente. El aumento en el Tween 20 permite la formación de micelas, las cuales solubilizarán parte del aceite, incrementando la viscosidad del sistema.

En la Tabla 2 se reporta el tamaño promedio de gota $D$ (3.2) y en la Figura 3 se muestra la distribución de tamaño de gota de las emulsiones O/W. Las emulsiones M1 a M10 presentaron tamaños de gota (D 3.2) en un rango entre 6.35 y $59.50 \mu \mathrm{m}$ a temperatura ambiente. El tamaño de gota más bajo $(6.35 \mu \mathrm{m})$ se obtuvo para la muestra con menor concentración de fase oleosa (5\%) y la concentración más alta de Tween 20 (30\%); lo cual está de acuerdo con la literatura, ya que una alta relación de surfactante a fase oleosa se requiere para producir pequeñas gotas usando métodos de baja energía (Piorkowski y McClements, 2014). Adicionalmente, una alta concentración de tensoactivo disminuye la tensión interfacial, lo cual conduce a un aumento del área superficial y por ende disminución del tamaño de gota. De otro lado, aunque no hay una tendencia perfecta, a menor tamaño de gota mayor es la viscosidad (Tabla 2).

En la industria es conocido que la estabilidad de las emulsiones y sus propiedades ópticas dependen del tamaño de gota (se recomienda que sea menor de $10 \mu \mathrm{m}$ para asegurar su estabilidad), ya que éste induce cambios en el área total interfacial que modifica el equilibrio de adsorción del surfactante (Rahn-Chique y Urbina-Villaba, 2017), y por ende la producción de fenómenos como separación gravitacional, floculación, coalescencia, entre otros (Piorkowski y McClements, 2014). Por esta razón, se seleccionaron las emulsiones de menor tamaño de gota (M1, M6 y M10) y se les adicionó goma xantana para incrementar su estabilidad. En la Tabla 2, se observa que el uso de goma xantana incrementó notablemente la estabilidad de las emulsiones, al disminuir el tamaño de gota a valores menores de $3.43 \mu \mathrm{m}$, pero no se logró llegar al rango de diámetro de gota reportado para microemulsiones, que son muy deseadas en la industria alimenticia por su transparencia.

En la Figura 3 se puede observar que las emulsiones M2, M3, M5, M7, M1A, M1B, M6A, M6B, M10A y M10B mostraron comportamiento monomodal, es decir que solo tienen un pico en la distribución de tamaño de partícula, lo cual es favorable porque esto usualmente provee mejor estabilidad a largo plazo (Piorkowski y McClements, 2014).

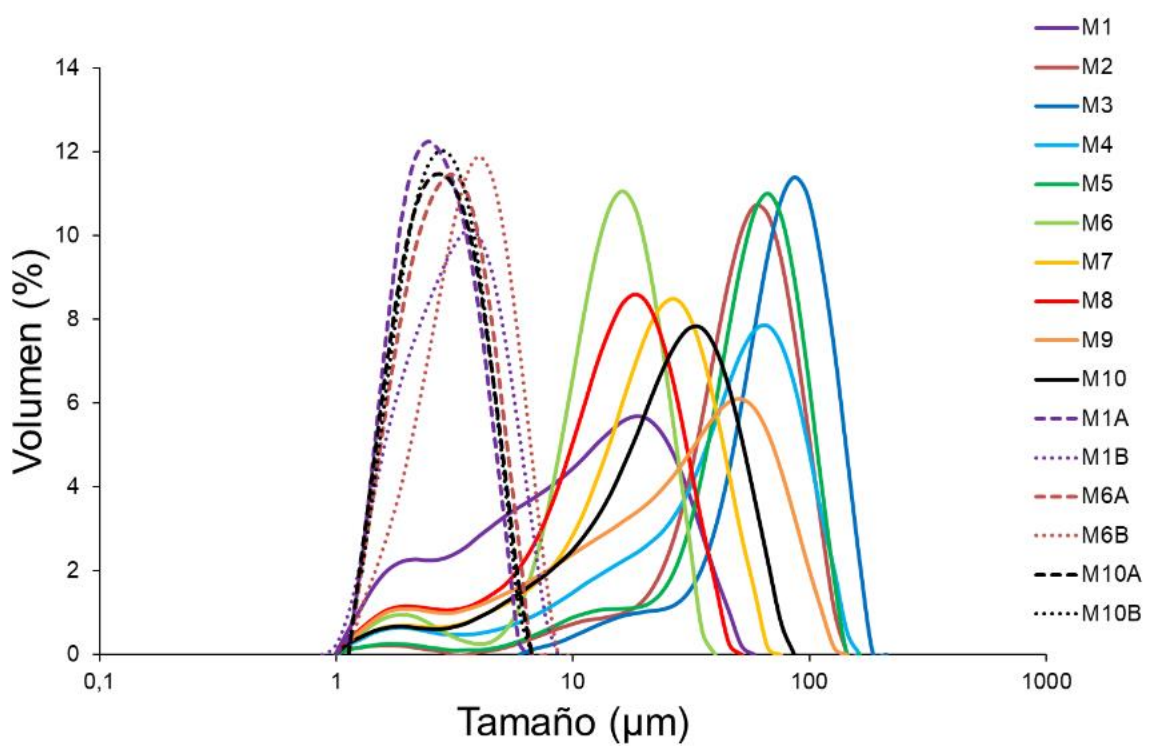

Fig. 3: Distribución de tamaño de gota para las emulsiones de extracto de uchuva (M1-M10B).

Para la medida del potencial $Z$, sólo se seleccionaron aquellas emulsiones con diámetro de gota menor a 10 $\mu \mathrm{m}$ (M1A, M6A, M6B, M10A y M10B), por ser las más estables (Tabla 3). 
Tabla 3: Parámetros obtenidos de la determinación del potencial $Z$ de las emulsiones de extracto de uchuva.

\begin{tabular}{c|c|c|c|c}
\hline Muestra & Potencial Z $(\mathrm{mV})$ & Desviación $(\mathrm{mV})$ & $\begin{array}{c}\text { Velocidad de } \\
\text { conteo }(\mathrm{kcps})\end{array}$ & $\begin{array}{c}\text { Relación de área } \\
\text { del pico }(\%)\end{array}$ \\
\hline M1A & -7.52 & \pm 2.88 & 52.0 & 100 \\
M6A & Pico 1:-12.1 & Pico 1: \pm 3.08 & Pico 1: 127.0 & Pico 1: 85.8 \\
& Pico 2: -11.3 & Pico 2: \pm 3.11 & Pico2: 127.0 & Pico2: 14.2 \\
M6B & -10.2 & \pm 4.26 & 446.0 & 100 \\
M10A & -10.2 & \pm 2.98 & 308.3 & 100 \\
M10B & -9.55 & \pm 2.96 & 744.6 & 100 \\
\hline
\end{tabular}

El potencial $Z$ es la carga general adquirida por partículas en un medio específico, su valor es un índice de la potencial estabilidad física de la emulsión. Si todas las partículas (gotas) tienen un potencial Z grande, ya sea positivo o negativo, ellas se repelerán entre sí y el sistema se considera estable; mientras más grande el potencial Z, mayor es la estabilidad del sistema. Las partículas (gotas) con un potencial Z entre $-10 \mathrm{mV}$ y +10 $\mathrm{mV}$ se consideran aproximadamente neutras, mientras que las partículas con potenciales $Z$ mayores de +30 $\mathrm{mV}$ o menores que $-30 \mathrm{mV}$ se consideran fuertemente catiónicas y fuertemente aniónicas, respectivamente. (Rahman et al., 2010; Hao et al., 2011). En el caso de las emulsiones obtenidas a partir de uchuva, se sugiere que la estabilidad conseguida está más relacionada con el aumento de la viscosidad que con la repulsión electrostática, debido a los componentes del sistema.

\section{Análisis de las propiedades sensoriales (colorimetría triestímulo)}

La evaluación del color a las emulsiones $\mathrm{O} / \mathrm{W}$ se realizó mediante colorimetría triestímulo, obteniendo los parámetros $L^{*}, a^{*}, b^{*}$, así como el chroma $\left(C^{*}\right)$ y el tono $\left(h^{*}\right)$ que se presentan en la Tabla 4 . Se observa que las emulsiones no presentaron colores intensos, teniendo en cuenta la baja proporción de fase oleosa que contiene los pigmentos (carotenoides). Analizando el parámetro $L^{*}$ (claridad) en comparación con la relación aceite/agua de cada emulsión, puede observarse que las emulsiones con mayor relación aceite/agua (M10, M2, M6 y M3) presentan un menor valor que las emulsiones con menor relación aceite/agua (M8, M4, M9, M5, M7); es decir, que estas últimas emulsiones se pueden considerar "más claras" debido al mayor contenido de agua. En cuanto al comportamiento del parámetro $C^{*}$ (croma), no se observa una tendencia definida; sin embargo, la emulsión M3 presentó el mayor valor de croma, siendo la emulsión con mayor concentración de fase oleosa. En cuanto a las emulsiones M1, M6 y M10 puede observarse que no existió diferencia significativa en el valor de $C^{*}$.

Tabla 4: Parámetros de colorimetría triestímulo y contenido de carotenoides totales para las emulsiones O/W y la fase oleosa obtenidas de uchuva. Letras iguales en la misma columna significa que no hay diferencias significativas $(p<0.5)$.

\begin{tabular}{c|c|c|c|c|c|c}
\hline Muestra & $L$ & $a^{*}$ & $b^{*}$ & $C a b^{*}$ & $h$ & $\begin{array}{c}\mathrm{mg} \beta- \\
\text { caroteno/g } \\
\text { emulsión }\end{array}$ \\
\hline M1 & $0.93^{\mathrm{a}}$ & $0.05^{\mathrm{c}}$ & $0.50^{\mathrm{a}}$ & $0.50^{\mathrm{a}}$ & $1.47^{\mathrm{a}}$ & 0.109 \\
M2 & $0.60^{\mathrm{a}}$ & $0.04^{\mathrm{c}}$ & $0.57^{\mathrm{a}}$ & $0.57^{\mathrm{a}}$ & $1.50^{\mathrm{b}}$ & 0.327 \\
M3 & $0.85^{\mathrm{a}}$ & $0.04^{\mathrm{c}}$ & $0.78^{\mathrm{b}}$ & $0.78^{\mathrm{b}}$ & $1.51^{\mathrm{b}}$ & 0.545 \\
M4 & $12.09^{\mathrm{b}}$ & $0.03^{\mathrm{b}}$ & $0.43^{\mathrm{a}}$ & $0.43^{\mathrm{a}}$ & $1.51^{\mathrm{b}}$ & 0.109 \\
M5 & $17.49^{\mathrm{c}}$ & $0.002^{\mathrm{a}}$ & $0.38^{\mathrm{a}}$ & $0.38^{\mathrm{a}}$ & $1.57^{\mathrm{c}}$ & 0.109 \\
M6 & $0.53^{\mathrm{a}}$ & $0.07^{\mathrm{d}}$ & $0.56^{\mathrm{a}}$ & $0.57^{\mathrm{a}}$ & $1.44^{\mathrm{a}}$ & 0.327 \\
M7 & $17.07^{\mathrm{c}}$ & $0.06^{\mathrm{d}}$ & $0.58^{\mathrm{a}}$ & $0.58^{\mathrm{a}}$ & $1.46^{\mathrm{a}}$ & 0.065 \\
M8 & $10.77^{\mathrm{b}}$ & $0.03^{\mathrm{b}}$ & $0.52^{\mathrm{a}}$ & $0.52^{\mathrm{a}}$ & $1.52^{\mathrm{b}}$ & 0.153 \\
M9 & $14.23^{\mathrm{c}}$ & $0.02^{\mathrm{b}}$ & $0.50^{\mathrm{a}}$ & $0.50^{\mathrm{a}}$ & $1.53^{\mathrm{b}}$ & 0.109 \\
M10 & $0.72^{\mathrm{a}}$ & $0.03^{\mathrm{b}}$ & $0.49^{\mathrm{a}}$ & $0.49^{\mathrm{a}}$ & $1.51^{\mathrm{b}}$ & 0.218 \\
Fase & & & & & & \\
oleosa & $89.00^{\mathrm{d}}$ & $2.00^{\mathrm{d}}$ & $49.00^{\mathrm{c}}$ & $48.65^{\mathrm{c}}$ & $1.52^{\mathrm{a}}$ & 2.180 \\
\hline
\end{tabular}

Análisis de las propiedades biofuncionales: contenido de carotenoides totales y actividad hipocolerestolémica

Inicialmente, se determinó el contenido de carotenoides totales mediante el método de estándar externo utilizando $\beta$-caroteno como estándar $\left(y=0.0004252 x+0.0000260, r^{2}=0,9944\right)$. El contenido de carotenoides en la fase oleosa fue de $2.180 \pm 0.228 \mathrm{mg} \beta$-caroteno/g muestra. Debido a la turbidez de las emulsiones no fue posible hacer las medidas de absorbancia directamente, así que se realizó un cálculo con base en el contenido de fase oleosa en cada emulsión (Tabla 1). Para verificar estos resultados, se graficó el contenido de $\beta$-caroteno de cada muestra frente al parámetro de color, croma $\left(C^{\star}\right)$ (Figura 4). Se puede observar que la emulsión M3, que presentó el mayor valor de croma, correspondió con un mayor contenido de carotenoides. Los carotenoides son colorantes naturales con propiedades biofuncionales (Mao et al., 2018). 


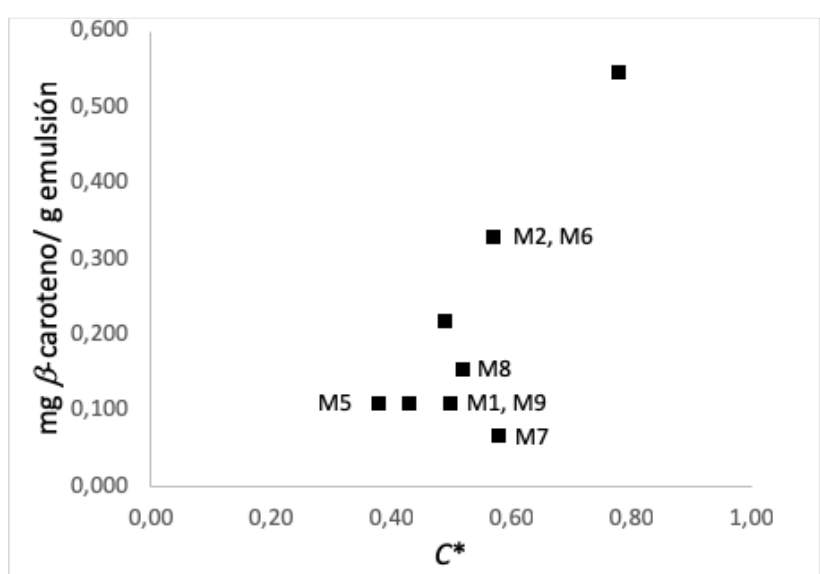

Fig. 4: Relación de contenido de $\beta$-caroteno vs. croma para las emulsiones $\mathrm{O} / \mathrm{W}$ de uchuva.

La actividad hipocolerestolémica se midió con un kit enzimático en donde se determinó el descenso en la absorbancia del NADPH por acción de la 3-hidroxi-3-metilglutaril-CoA reductasa (HMG-CoA) en presencia del sustrato HMG-CoA. Esta enzima es la que controla la velocidad de la ruta del mevalonato, mediante la cual se produce colesterol a partir de acetil CoA. Los fármacos que reducen el colesterol, conocidos como las estatinas, inhiben dicha enzima. En la Fig se presentan los resultados obtenidos para el blanco, la enzima (control no inhibitorio), la pravastatina (control inhibitorio), la fase acuosa de uchuva (extracto liofilizado de uchuva, redisuelto en agua y filtrado) y la fase oleosa de uchuva. Se comprobó que la enzima estaba funcionando y que la fase oleosa tenía más actividad hipocolerestolémica que la fase acuosa.

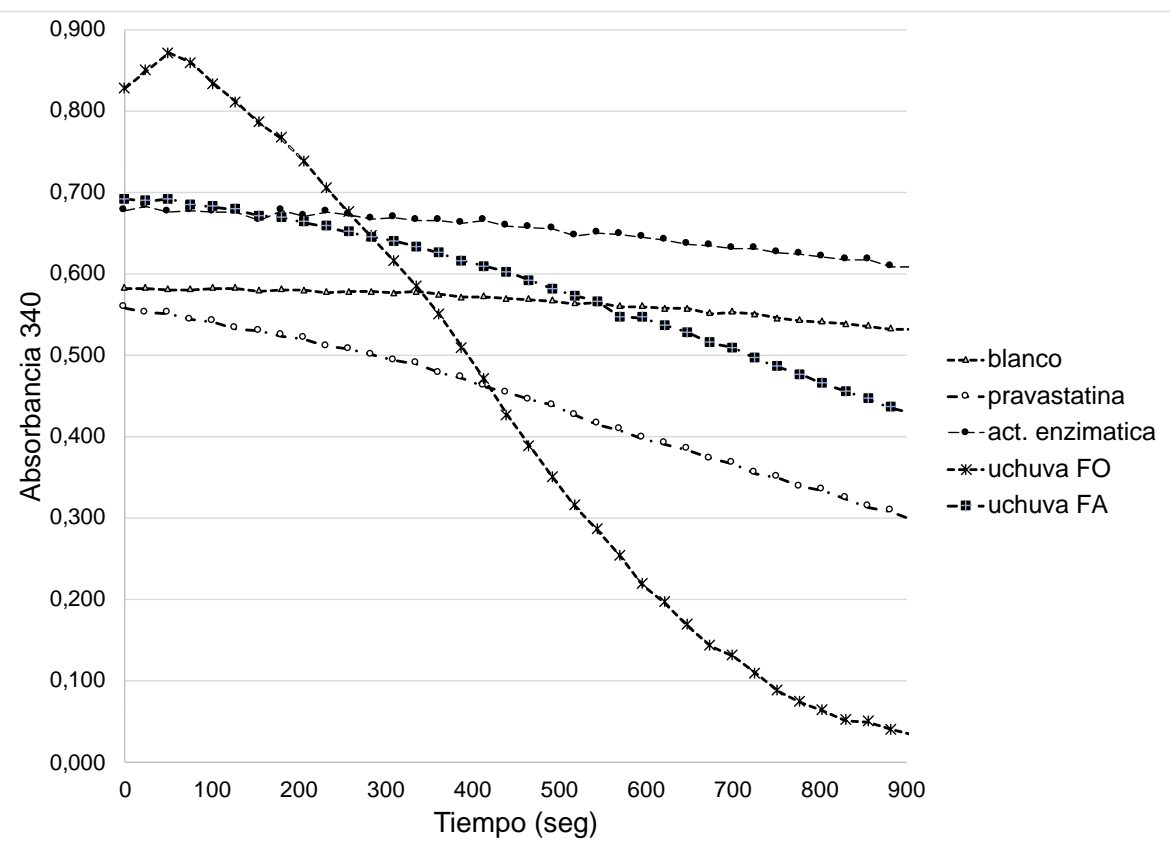

Fig. 5: Variación del parámetro absorbancia en el tiempo para las muestras utilizadas en el ensayo de actividad antihipercolerestolémica.

Más significativo aun, la fase oleosa de uchuva tiene una actividad mayor que la del control Pravastatina. En este orden de ideas el presente trabajo tiene una justificación alta, ya que se pretendió obtener una emulsión O/W para proporcionar un ingrediente bioactivo de uchuva a la industria alimenticia debido a la presencia de fitoquímicos.

\section{CONCLUSIONES}

Se confirmó que el método de emulsificación espontánea (SE) es un método relativamente sencillo y efectivo para la producción de emulsiones preparadas a partir del extracto de uchuva liofilizada en aceite de girasol, el cual exhibió actividad hipocolerestolémica in vitro. La medida del potencial Z permitió definir cuáles fueron las emulsiones más estables, después de la adición de goma xantana que incrementó la viscosidad, disminuyó el tamaño de gota al impedir la coalescencia y formación de gotas grandes y por ende mejoró la 
estabilidad. Las emulsiones con menor contenido de fase oleosa fueron más estables, sin embargo, la fase oleosa es rica en carotenoides y mostró una actividad hipocolerestolémica mayor que el control pravastatina.

\section{AGRADECIMIENTOS}

Los autores agradecen el apoyo financiero del Fondo Nacional de Financiamiento para la Ciencia, la Tecnología y la Innovación, Francisco José de Caldas, contrato No. 0459 - 2013, Red Nacional para la Bioprospección de Frutas Tropicales-RIFRUTBIO y de la División de Investigación-Sede Bogotá, Universidad Nacional de Colombia. También agradecen a la Dra.Tatiana Cuadrado por el apoyo en la medida de la actividad antihipercolerestolémica y al químico M.Sc. Mauricio García de la empresa Nabla Ciencia en Movimiento, por las medidas del potencial $Z$ y las discusiones concernientes a estabilidad de emulsiones.

\section{REFERENCIAS}

Abd-Allah, F.I., H. M. Dawaba y A. M. S. Ahmed, Development of a microemulsion-based formulation to improve the availability of poorly water-soluble drug, Drug Discov. Ther., 4(4), 257-266 (2010)

Agronet, Base de datos de cifras agropecuarias en Colombia. Consultado en julio (2018)

Analdex, Empiezan a consolidarse exportaciones de Uchuva a Estados Unidos. Consultado en julio 2018

Bernal, C.-A., M. Aragón e Y. Baena, Dry powder formulation from fruits of Physalis peruviana L. standardized extract with hypoglycemic activity, doi: 10.1016/j.powtec.2016.07.008, Powder Technol., 301, 839-847 (2016)

Brunchi, C-E., M. Bercea, S. Morariu y M. Dascalu, Some properties of xanthan gum in aqueous solutions: Effect of temperature and pH, doi: 10.1007/s10965-016-1015-4, J. Polym. Res., 23, 1-8 (2016)

Dluzewska, E., A. Stobiecka y M. Maszewska, Effect of oil phase concentration on rheological properties and stability of beverage emulsions, Acta Sci. Pol., Technol. Aliment., 5(2), 147-156 (2006)

El-Ashhab, F., L. Sheha y H. Abo-Eisa, Polyelectrolytic behaviour of photodegradable xanthan solutions. Part II. Conductivity change in aqueous solution, Journal of Science and Its Applications, 2(1), 77-82 (2008)

Ghosh, V., A. Mukherjee y N. Chandrasekaran, Ultrasonic emulsification of food-grade nanoemulsion formulation and evaluation of its bactericidal activity, doi: doi.org/10.1016/j.ultsonch.2012.08.010, Ultrason. Sonochem., 20(1), 338-344 (2013)

Giraldo, G. I., C. D. Cruz y N. R. Sanabria, Propiedades físicas del jugo de uchuva (Physalis peruviana) clarificado en función de la concentración y la temperatura, doi: 10.4067/S0718-07642017000100013, Inf. Tecnol., 28(1), 133-142 (2017)

Hao, J., X. Fang y otros cinco autores, Development and optimization of solid lipid nanoparticle formulation for ophthalmic delivery of chloramphenicol using a Box-Behnken design, doi: 10.2147/IJN.S17386, Int. J. Nanomedicine, 6, 683-692 (2011) ICONTEC, Norma Técnica Colombiana NTC 4580. Frutas Frescas. Uchuva. Especificaciones. Bogotá (1999)

Komaiko, J. S. y D. J. McClements, Formation of food-grade nanoemulsions using low-energy preparation methods: A review of available methods, doi: doi.org/10.1111/1541-4337.12189, Compr. Rev. Food Sci. Food Saf., 15(2), $331-352$ (2016)

Mao, L., D. Wang, F. Liu e Y. Gao, Emulsion design for the delivery of $\beta$-carotene in complex food systems, Crit. Rev. Food Sci. Nutr., 58(5), 770-784 (2018)

Martínez, J., Microencapsulación de pigmentos carotenoides en harina de residuos de chontaduro (Bactris gasipaes) mediante secado por atomización: Una alternativa de colorante natural para productos panificados. Tesis de Maestría en ingeniería agroindustrial, Universidad Nacional de Colombia, Palmira (Valle), Colombia (2016)

McClements, D., Food Emulsions: Principles, Practices, and Techniques. C. Press, Boca Raton, FL, EUA (2005)

Osorio, C., M. E. Schreckinger y otros cuatro autores, Golden berry and selected tropical (açai, acerola, and maqui) juices. En F. Shahidi \& C. Alasalvar (Eds.), Handbook of Functional Beverages and Human Health, 251-269. Taylor \& Francis Group, Boca Raton, FL, Estados Unidos (2016)

Piorkowski, D.T. y D.J. McClements, Beverage emulsions: Recent developments in formulation, production, and applications, doi: doi.org/10.1016/j.foodhyd.2013.07.009, Food Hydrocoll., 42(1), 5-41 (2014)

Ramadan, M. F., N. A. Hassan, R. M. Elsanhoty y M. Z. Sitohy, Goldenberry (Physalis peruviana) juice rich in healthbeneficial compounds suppresses high-cholesterol diet-induced hypercholesterolemia in rats, doi: doi.org/10.1111/j.17454514.2012.00669.x, J. Food Biochem., 37(6), 708-722 (2013)

Ramírez, L., Desarrollo y caracterización de granulados enriquecidos en aroma y color a partir de frutas de uchuva (Physalis peruviana L). Tesis de pregrado en química farmaceútica. Universidad Nacional de Colombia, Bogotá, Colombia (2009)

Rahman, Z., A.S. Zidan, M.J. Habib y M.A. Khan, Understanding the quality of protein loaded PLGA nanoparticles variability by Plackett - Burman design, doi: 10.1016/j.jpharm.2009.12.040, Int. J. Pharm., 389(1-2), 186-194 (2010)

Rahn-Chique, K. y G. Urbina-Villalba, Dependence of emulsion stability on particle size: relative importance of drop concentration and destabilization rate on the half lifetimes of $\mathrm{O} / \mathrm{W}$ nanoemulsions, doi: 10.1080/01932691.2016.1149715, J. Disper. Sci. Technol., 38(2), 167-179 (2017) 
Schramm, L. L., Emulsions, Foams, and Suspensions. Fundamentals and Applications. WILEY-VCH Verlag GmbH \& Co. KGaA, Weinheim, Alemania (2005)

Siró, I., E. Kápolna, B. Kápolna y A. Lugasi, Functional food, Product development, marketing and consumer acceptanceA review, doi: 10.1016/j.appet.2008.05.060, Appetite, 51(3), 456-467 (2008)

Vieira da Silva, B., J. C. M. Barreira y M. B. P. P. Oliveira, Natural phytochemicals and probiotics as bioactive ingredients for functional foods: Extraction, biochemistry and protected-delivery technologies, doi: doi.org/10.1016/j.tifs.2015.12.007, Trends Food Sci. Technol., 50, 144-158 (2016)

Wrolstad, R.E., T. E. Acree y otros seis autores, Detection and measurement of carotenoids by UV/VIS spectrophotometry. En Handbook of Food Analytical Chemistry. Pigments, Colorants, Flavors, Texture and Bioactive Food Components. John Wiley \& Sons, Hoboken, NJ, Estados Unidos (2005) 